\title{
Radioprotección de la médula espinal mediante la hormona de crecimiento (GH). Estudio experimental en ratas
}

\author{
A. Isla; M. Budke; A. García-Grande; A. Gómez de la Riva; C. Morales y J. Rey
}

Servicios de Neurocirugía y Radioterapia. Hospital Universitario La Paz. Madrid.

\section{Resumen}

La radioterapia empleada en el tratamiento de los tumores o malformaciones vasculares del SNC presenta riesgo de causar lesiones en los tejidos adyacentes.

El objetivo de nuestro estudio es demostrar las alteraciones provocadas por la radioterapia en la medula espinal de ratas a altas dosis y el valorar el efecto protector de la hormona de crecimiento (GH). El estudio experimental fue realizado con dos grupos de ratas Wistar. Grupo A (grupo control): Constó de 10 ratas que se irradiaron con $30 \mathrm{~Gy}$ en la medula espinal. Grupo B: Fue un grupo de 10 ratas irradiadas con 30 Gy a las que se administró $2 \mathrm{mg} / \mathbf{k g} /$ día de GH tres días previos a la radioterapia, el día de la radioterapia, y dos días después. A los 14 días de la radioterapia fueron perfundidas mostrando el estudio histológico de la médula espinal de las ratas tratadas sólo con radioterapia de forma intensa, hemorragias y trombosis de capilares. Al grupo a las que se administró GH mostró una importante disminución de las lesiones.

En resumen la radioterapia administrada en dosis de 30 Gy causa cambios morfológicos como edema lesión del endotelio vascular, necrosis, hemorragias, exudado inflamatorio. La dosis de $2 \mathrm{mg} / \mathrm{kg} /$ día ejerce un efecto protector en la medula espinal de ratas tras la administración de radioterapia disminuyendo el daño macro y microscópico en las ratas estudiadas.

PALABRAS CLAVE: Hormona de crecimiento. Medula espinal. Radionecrosis.

Protective effects of the growth hormone (GH) on the irradiated spinal cord in rats

\section{Summary}

Radiotherapy or irradiation of SNC AVM's or tumors also presents a high risk for provoking lesions

Recibido; 4-03-06. Aceptado: 12-07-06 in adjacent surrounding tissue.

The objective of our study is to demonstrate radiotherapy induced alterations in a rat spinal cord model and evaluate the protective effect of Growth Hormone (GH) on rats exposed to high radiotherapy doses.

The experimental study employed two groups of Wistar rats: Group A (control group):10 rats, which received $30 \mathrm{~Gy}$ at the spinal cord . Group B: 10 rats, these animals received $30 \mathrm{~Gy}$ and dose of $2 \mathrm{mg} / \mathrm{kg} / \mathrm{day}$ GH. Growth hormone administration was begun three days before radiotherapy and continued until two days after radiotherapy for a total of six days. At 14 days postradiotherapy, all the rats were sacrificed and the spinal cord extracted immediately. Hematoxyline-eosine histologic studies showed that control animals only exposed to radiotherapy had severe alterations with hemorrhage and vacuolisation of the entire irradiated segment while these alterations were much less severe in the GH-treated group.

In conclusion, 30 Gy irradiation produced morphological changes including vascular endothelial oedema, necrosis, hemorrhage, and inflamantory exudates. A $2 \mathrm{mg} / \mathrm{kg} /$ day dose of GH protected the rat spinal cord against the noxious effects of the radiotherapy, decreasing the clinical, macro and microscopic damage in the treated animals.

KEY WORDS: Growth hormone. Spinal cord. Radionecrosis.

\section{Introducción}

La irradiación empleada en la terapéutica radical o en el tratamiento paliativo de los tumores o malformaciones vasculares del sistema nervioso central (SNC) presenta un alto riesgo de causar lesiones en los tejidos neurales perilesionales sanos y también al radiar patologías cercanas al sistema nervioso ${ }^{8}$.

Los daños producidos por la irradiación del SNC se consideran el principal factor que limita el empleo de este tipo de tratamiento en radioterapia, ya que existe una 
íntima asociación entre el grado de las alteraciones producidas y factores como la dosis empleada y la duración del tratamiento radioterapéutico.

El SNC está constituido básicamente por neuronas, células de la glía (oligodendrogliocitos, astrocitos y microglia), células ependimarias y células del endotelio vascular. Cada tipo celular desempeña una tarea específica en el funcionamiento del SNC y la respuesta a la irradiación se establece también por las características histológicas de cada tipo celular. Las lesiones originadas por la irradiación del SNC afectan principalmente a las células que componen la glía y a las células del endotelio vascular ${ }^{16}$.

Entre las células de la glía, los oligodendrogliocitos (cuya función es la formación y el mantenimiento de la vaina de mielina axonal) son las células con mayor grado de afectación, en contraste con las neuronas, que por su alto grado de diferenciación y su escasa proliferación celular se consideran radiorresistentes ${ }^{11}$. Los principales cambios morfopatológicos producidos por la irradiación en el SNC consisten en una desmielinización producida por la muerte de los oligodendrocitos, asociada a áreas de necrosis en la sustancia blanca, que posteriormente ocasionan la formación de cavidades (malacias) y atrofia cerebral ${ }^{16}$.

La muerte celular observada a consecuencia de la irradiación ocurre básicamente por dos mecanismos: apoptosis y necrosis celular. La apoptosis es un proceso de muerte celular programada por el cual el ADN sufre una rotura de su cadena y una degeneración de su estructura molecular a consecuencia de un daño cromosómico irreparable. La necrosis es un proceso distinto: la irradiación causa la muerte celular por el hinchamiento de la célula y de sus organelas, con la consiguiente ruptura de la membrana celular antes de la degradación del ADN ${ }^{4,15}$. Estudios recientes han demostrado que la GH (hormona del crecimiento) desempeña una función protectora en ratas tratadas con irradiación; se ha observado que protege las células de la mucosa intestinal de los efectos de la radioterapia, disminuyendo el número de células apoptóticas en la cripta intestinal ${ }^{14,18}$. La GH es una hormona secretada por el lóbulo anterior de la hipófisis y desempeña una importante función en el desarrollo del SNC. Actúa por medio de sus receptores en el SNC, produciendo una proliferación de las células gliales y la maduración de la glía y de las neuronas; también contribuye a la formación de la vaina de mielina ${ }^{10,12}$. En los cultivos de células de cortezas de embriones de ratas, la GH causa una acentuada proliferación celular y, posiblemente, desempeña una función protectora actuando en sus receptores específicos o indirectamente por medio de IGFI (del inglés insulin-like growth factor $)^{10}$. El objetivo del presente estudio es valorar el posible efecto protector de la $\mathrm{GH}$ en la medula espinal de la rata a dosis alta, con respecto a otro grupo donde no se administró la hormona.

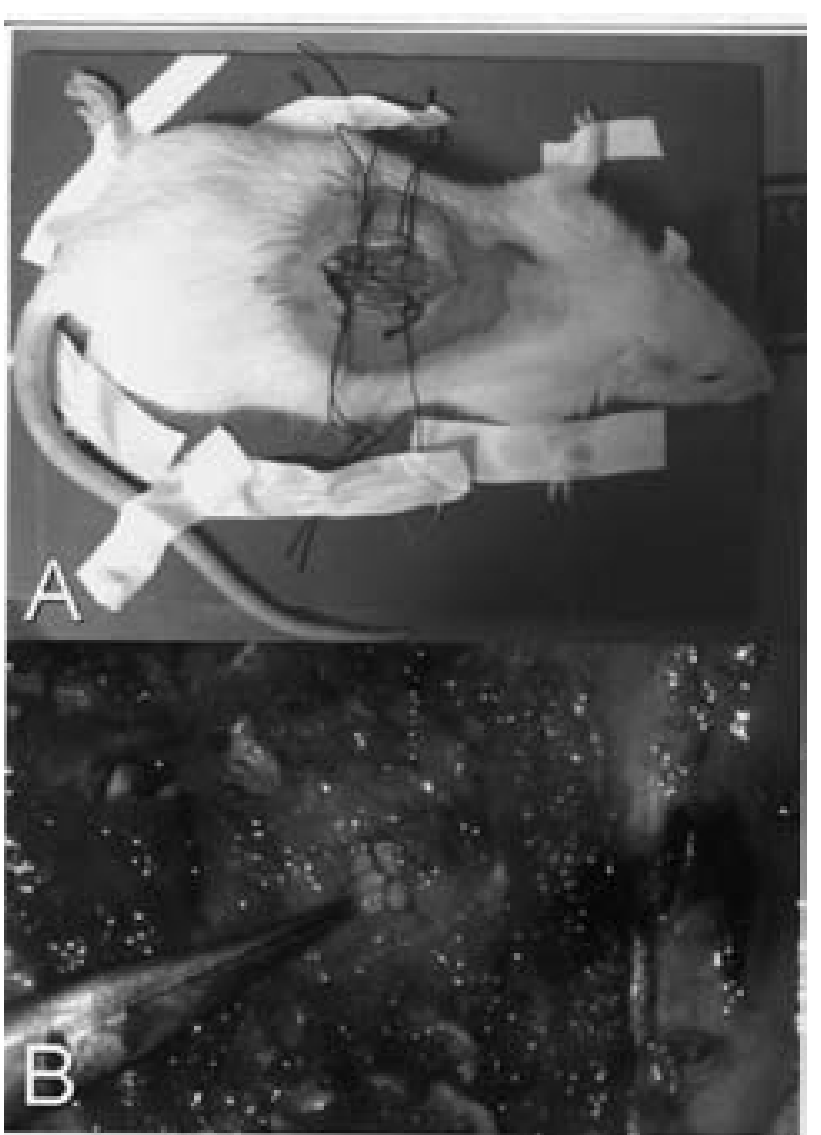

Figura 1. (A y B): Laminectomias con extirpación del arco posterior de D6 y D7 y exposición de la dura madre.

\section{Materiales y métodos}

Utilizamos ratas Wistar de 350 - 550 gr de peso. En todas ellas se realizó el siguiente procedimiento quirúrgico: anestesia intraperitoneal con la combinación de $\operatorname{Ketolar}^{\mathrm{R}}$ (0.5cc), Valium ${ }^{\mathrm{R}}$ (0.4 cc) y Atropina (0.2 cc) ; previa asepsia con solución yodada y fijación del animal en un aparato de estereotaxia, se realizó una incisión longitudinal medial en la piel de la región espinal torácica con la consiguiente exposición del cordón espinal. Se expusieron dos segmentos entre T5 y T10 y se practicó una incisión longitudinal de $2 \mathrm{~cm}$ en la línea media en la región dorsal con disección subperióstica con microscopio. A continuación se realizaron laminectomías con extirpación del arco posterior de D6 y D7 y exposición de la dura madre (Fig. 1 A y B).

Como fuente de irradiación se utilizó un aparato de irradiación por contacto tipo Roetgen, con un aplicador C2 de 10 mm que por visión directa se dirigió a la diana (Fig. 2). La dosis a utilizar fue la necesaria para producir edema medular (y/o radionecrosis) en el menor tiempo posible. La dosis encontrada para producir este tipo de lesión, según un protocolo previo diseñado en nuestro servicio por el método de dosis creciente, fue de 30.000 cGy, con 


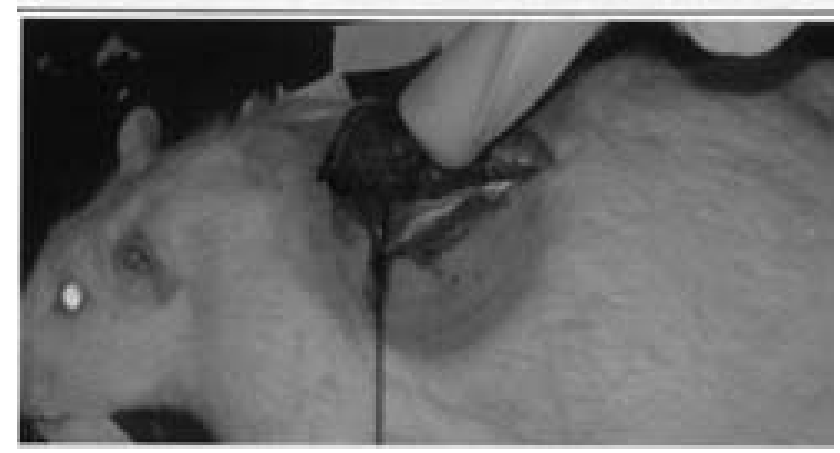

Figura 2: Administración de radioterapia por contacto tipo Roetgen, con un aplicador C2 de $10 \mathrm{~mm}$ que por visión directa se dirigió a la diana.

un tiempo de exposición por contacto de 9 minutos con 74 segundos, y con una tasa de dosis de 5133 cGy / min. La lesión causada por dicha radiación se desarrolló en menos de una semana de seguimiento.

El trabajo constó de 2 grupos principales:

Grupo A (control): Fue de 10 ratas en las que sólo fueron tratadas con radioterapia medular a una dosis de 30 Gy

Grupo B: También formado por 10 ratas en las que se realizó un tratamiento con radioterapia medular y con la GH a una dosis de $2 \mathrm{mg} / \mathrm{kg} /$ día de forma subcutánea. La dosis de administración de la hormona GH (Genotonorm ${ }^{\circledR}$ de Pfeizer) fue de tres días previo a la radioterapia, en el día de la radioterapia y dos días posteriores a la radioterapia.

A los 14 días después de la radiación, todas las ratas fueron sacrificadas y prefundidas intracardicamente con paraformaldehído al 4\% , teniendo especial cuidado en extraer la medula espinal para su inclusión en parafina. Seguidamente se realizaron cortes sagitales para estudio de los cambios morfológicos con tinción de hematoxilinaeosina con el objetivo de comparar en los dos grupos el área de edema y/o radionecrosis, reacción glial, y características vasculares del tejido cerebral irradiado. El grado de lesión histológica se realizó según la extensión de la lesión histológica y lo dividimos en 5 grados lesionales: muy leve corresponde de 0-2 mm, leve de 2-4 mm, moderado de 4-6 $\mathrm{mm}$, grave de $6-8 \mathrm{~mm}$ y muy grave mayor de $8 \mathrm{~mm}$. Una vez realizada las mediciones se realizaron estudio estadístico para observar la diferencia entre ambos grupos.

\section{Resultados}

La extensión de la lesión se midió con ayuda del microscopio utilizando un ocular milimetrado con objetivo $2 \mathrm{x}$ valorándose la extensión de la lesión en milímetros en los cortes longitudinales de las médulas (Tabla 1).

Los resultados de los estudios realizados con hematoxlina-eosina según la valoración realizada (5 grados

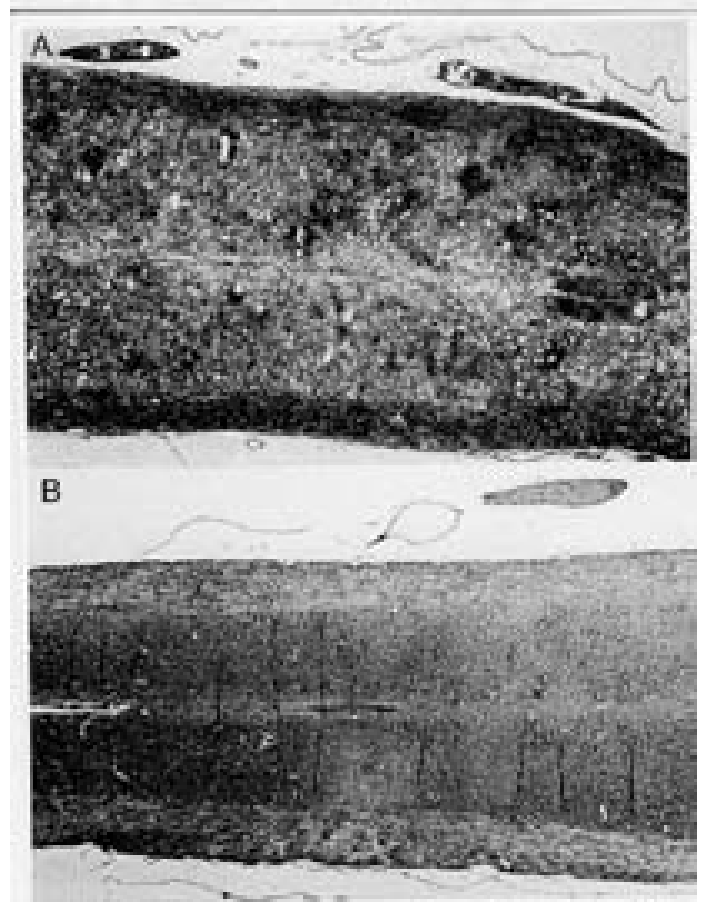

Figura 3. (A y B): Ejemplos de los estudios realizados en la medula espinal (cotes coronales) con Hematoxlina-eosina (3x) 14 días de la administración de la Radioterapia (30 Gy). A. Imagen de médula espinal de rata a la que se administró radioterapia y no se le protegió con GH. Se observa un grado lesional grave con graves alteraciones histológicas con hemorragias y vacuolización de todo el segmento donde se administró radioterapia (Grado 5 de lesión). B Corte histológico de médula espinal de rata, a la que además de la radioterapia (misma dosis) se administró $\mathrm{GH}$, no apreciándose lesiones de importancia (Gado 1 de lesión).

lesionales) en muy leve, leve, moderado, grave y muy grave mostraron que en el grupo donde sólo se administró radioterapia la mayoría de las ratas presentaban graves alteraciones histológicas con hemorragias y vacuolización de todo el segmento donde se administró radioterapia, observándose afectación leve en 3 casos, moderada en 1 caso, grave en 1 caso y muy grave en 5 casos (Fig. 3 A y B). En el grupo en el que se administró radioterapia y se trató con GH se evidenció una disminución de las lesiones, apreciándose 6 casos muy leves de afectación, 2 casos leves, 1 caso moderado y 1 caso grave Tabla 2).

El análisis estadístico se realizó comparando la media de la extensión de la lesión de los dos grupos con la T-Students. En dicho análisis se observó que en el grupo GH negativo (GH-) presentó la extensión de la lesión con una media de 7,90 mm con desviación estándar de 4,067, y en el grupo tratado con la GH (GH+) una media de 2,4 mm con desviación estándar de 1,35. Comparando los dos grupos la diferencia estadística fue significativa $\mathrm{p}<0,005$ (Tabla 3 ). 
Tabla 1

Extensión lesional en médula espinal post-radioterapia

\begin{tabular}{|c|c|l|}
\hline $\mathrm{N}^{\circ}$ Rata & $\begin{array}{c}\text { Extensión } \\
\text { lesional } \\
\text { (Sin } \mathrm{GH})\end{array}$ & $\begin{array}{l}\text { Extensión } \\
\text { lesional } \\
\text { (Con GH) }\end{array}$ \\
\hline 1 & $15 \mathrm{~mm}$ & $5 \mathrm{~mm}$ \\
\hline 2 & $10 \mathrm{~mm}$ & $1 \mathrm{~mm}$ \\
\hline 3 & $10 \mathrm{~mm}$ & $4 \mathrm{~mm}$ \\
\hline 4 & $3 \mathrm{~mm}$ & $7 \mathrm{~mm}$ \\
\hline 5 & $9 \mathrm{~mm}$ & $2 \mathrm{~mm}$ \\
\hline 6 & $4 \mathrm{~mm}$ & $1 \mathrm{~mm}$ \\
\hline 7 & $12 \mathrm{~mm}$ & $3 \mathrm{~mm}$ \\
\hline 8 & $5 \mathrm{~mm}$ & $2 \mathrm{~mm}$ \\
\hline 9 & $3 \mathrm{~mm}$ & $1 \mathrm{~mm}$ \\
\hline 10 & $8 \mathrm{~mm}$ & $2 \mathrm{~mm}$ \\
\hline
\end{tabular}

Tabla 2

Extensión lesional en médula espinal post-radioterapia

\begin{tabular}{|c|l|l|c|c|}
\hline $\mathrm{N}^{\circ}$ & $\begin{array}{l}\text { Extensión } \\
\text { lesional }\end{array}$ & $\begin{array}{l}\text { Grado } \\
\text { lesional }\end{array}$ & Sin GH & Con GH \\
\hline 1 & $0-2 \mathrm{~mm}$ & Muy leve & & 6 \\
\hline 2 & $2-4 \mathrm{~mm}$ & leve & 32 & \\
\hline 3 & $4-6 \mathrm{~mm}$ & moderado & 11 & \\
\hline 4 & $6-8 \mathrm{~mm}$ & grave & 11 & \\
\hline 5 & $>8 \mathrm{~mm}$ & Muy grave & 5 & \\
\hline
\end{tabular}

\section{Discusión}

La irradiación empleada en el tratamiento de los tumores y malformaciones vasculares del SNC puede causar importantes alteraciones en los tejidos adyacentes ${ }^{8}$. En el tratamiento con radiocirugía se administra una dosis máxima de radioterapia en un área reducida de tejido nervioso que puede implicar lesiones en áreas que rodean a estos procesos.

En el ámbito celular, las principales alteraciones producidas son la muerte de las células del endotelio vascular, de las células de la glía y, en dosis más elevadas, también de las neuronas ${ }^{1,3,9,12,13,16}$.

Tradicionalmente, la muerte celular de las células expuestas a la irradiación se ha atribuido a los fenómenos de apoptosis y necrosis ${ }^{16}$. La muerte por apoptosis es un mecanismo complejo en el cual la célula, sin posibilidades de reparar adecuadamente el daño en la cadena de ADN causado por la irradiación, entra en un proceso de muerte autoprogramada; este tipo de muerte sería una forma activa de "suicidio", en el cual el citoplasma y el núcleo celular, tras sufrir un proceso de degeneración, quedarían condensados en la periferia de la célula ${ }^{7}$. Por otra parte, el daño vascular producido por la afectación del endotelio contribuiría a la disminución de la vascularización e indirectamente a daño isquémico con muerte celular ${ }^{1,3}$.

Estudios microscópicos realizados en células de cortezas de ratas jóvenes tratadas con radioterapia demuestran la existencia de apoptosis celular en dosis de irradiación entre 0,4 y $4 \mathrm{~Gy}^{7,11}$. Las interacciones entre las neuronas y la neuroglia ocurren por medio de varias moléculas señalizadoras como las citoquinas, iones y metabolitos ${ }^{6,11}$.

Respecto a la interacción de las células del SNC irradiadas se supone que una de las funciones de los astrocitos sería la protección de las neuronas, evitando su muerte. Una posible hipótesis -según algunos estudios experimentales realizados- sería que los astrocitos, por su capacidad de captar el glutamato extracelular, protegerían las neuronas

Tabla 3

Resultado estadístico de la extensión lesional en médula espinal post-radioterapia

\begin{tabular}{|c|c|c|c|c|c|c|}
\hline \multirow{3}{*}{ Extensión de lesión en mm } & Grupo & \multicolumn{2}{|c|}{$\mathrm{N}^{\mathrm{o}}$} & \multicolumn{2}{|c|}{ Media } & Std. Desviación \\
\hline & GH Negativo & 1 & 0 & 7,9 & 0 & 4,067 \\
\hline & GH Positivo & 1 & 0 & 2,4 & 0 & 1,350 \\
\hline
\end{tabular}

T-Student. $\mathrm{P}<0.005$ 
de la toxicidad producida por la radioterapia.

En los cultivos tratados con irradiación y con GH observamos un número inferior de células apoptóticas 24 horas después del tratamiento con radioterapia ${ }^{7}$. La rotura de la cadena de ADN, causada por la radioterapia, promueve la apoptosis celular siempre que la doble hélice no se repara adecuadamente ${ }^{2}$. Actualmente es conocido que el tejido nervioso dispone de receptores de $\mathrm{GH}^{5,17}$ y que la $\mathrm{GH}$ puede actuar a nivel del SNC. Se conoce que GH ejerce una acción mitogénica induciendo a un aumento en la expresión del péptido cmyc, un marcador del ciclo celular que sincroniza la transición de la fase GO-G1 del ciclo celular a la fase $\mathrm{S}$, en la síntesis del ADN. Esta sincronización de las células en la misma fase (fase S) del ciclo celular podría hacerlas más radiorresistentes y posiblemente sería uno de los mecanismos protectores ejercidos por la $\mathrm{GH}^{18}$.

Otro probable mecanismo de protección sería la proliferación de las células gliales causada por la GH, debido al papel fundamental ejercido por la glía en el intercambio de iones entre las células de la corteza y el espacio intercelular ${ }^{6}$. En los cultivos sometidos a radioterapia, la glía ejerce una función radioprotectora captando el glutamato extracelular y disminuyendo su toxicidad para las células presentes en los cultivos ${ }^{7,11}$. Esta acción probablemente se limita a las células que presentan receptores para la GH en sus membranas, con el fin de que esta hormona pueda ejercer su efecto protector. En este sentido, la GH, provocando proliferación de las células gliales, protegería a todas las células de la toxicidad del glutamato extracelular. También se ha observado que la GH tiene un efecto vasodilatador y de protección del endotelio vascular ${ }^{1}$ por lo que influiría en la protección del daño que podría producirse por la irradiación.

En nuestro estudio hemos comprobado que la $\mathrm{GH}$ administrada a ratas cuando éstas reciben dosis altas de irradiación a nivel de la médula espinal, las preserva de presentar lesiones preferentemente de tipo vascular como histológicamente se ha demostrado con hematoxilina-eosina con respecto a un grupo control en donde no se administró radioterapia. En el análisis estadístico se observó que en el grupo sin GH presentó la extensión de la lesión con una media de 7,90 mm con desviación estándar de 4,067 y en el grupo tratado con la GH una media de 2,4 mm con desviación estándar de 1,35. Comparando los dos grupos la diferencia estadística fue significativa $\mathrm{p}<0,005$.

En resumen, creemos que la GH es útil como radioprotector y puede tener diversas aplicaciones, como en la radiocirugía o la radioterapia, donde se administra una dosis elevada de radiación que puede implicar lesiones del sistema nervioso en las áreas adyacentes al proceso a tratar como malformaciones vasculares o tumores.

La radioterapia administrada a la médula espinal de la rata a dosis de 30 Gy produce cambios morfológicos como edema lesión del endotelio vascular, necrosis, hemorragias, exudado inflamatorio.

La hormona de crecimiento en una dosis de $2 \mathrm{mg} / \mathrm{kg} /$ día subcutánea ejerce un efecto protector en la médula espinal de ratas tras la administración de radioterapia disminuyendo el daño macro y microscópico en las ratas estudiadas.

\section{Bibliografía}

1. Ariznavarreta, C., Castillo, C., Segovia, G., Mora, F., Azcoitia I, Tresguerres, J.A.: Growth hormone and aging. Homo. 2003; 54: 132-141.

2. Borovitskaya, A.E., Evtushenko, V.I., Sabol, S.L.: Gammaradiationinduced cell death in the fetal brain possesses molecular characteristics of apoptosis and is associated with specific messenger RNA elevations. Mol Brain Res 1996; 35: 19-30.

3. Dewey, W.C., Ling, C.C., Meyn, R.E.: Radiationinduced apoptosis: relevance to radiotherapy. Int J Radiat Oncol Biol Phys 1995; 33: 781-795.

4. Fuks, Z., Persaud, R.S., Alfieri, A., McLoughlin, M., Ehleiter, D., Scwartz, J.L., et al.: Basic fibroblast growth actor protects endothelial cells against radiationinduced programmed cell death in vitro and in vivo. Cancer Res 1994, 54: 2582-2590.

5. García-Aragón, J., Lobie, P.E., Muscat, G.E., Gobius, K.S., Norstedt, G., Waters M.J.: Prenatal expression of the growth hormone $(\mathrm{GH})$ receptor/binding protein in the rat: a role for GH in embryonic and fetal development? Development 1992; 114: 869-876.

6. Haimovitz-Friedman, A., Vlodaysky, I., Chadhuri, A., Witte, L., Fuks, Z.: Autocrine effects of fibroblast growth factor in repair of radiation damage in endothelial cells. Cancer Res 1991; 51: 2552-2558.

7. Isla, A., Budke, M., Cacicedo, L., García Grande, A., Vázquez Rodríguez, I., Miguel, E., Pérez Alvarez, M.: El efecto protector de la hormona de crecimiento en cultivos de células del sistema nervioso central. Rev Neurol 2002; 34: 208-211.

8. Lengyel, Z., Rékó, G., Matjtényi, K., Pisch, J., Csornai, M., Lesznyák, Trón, L., Esik, O.: Autopsy verifies demyelination and lack of vascular damage in partially reversible radiation myelopathy Spinal Cord 2003; 41, 577-585.

9. Li, Y.Q., Jay, V., Wong, C.S.: Oligodendrocytes in the adult rat spinal cord undergo radiationinduced apoptosis. Cancer Res 1996; 56: 5417-5422.

10. Lobie, P.E., García Aragón, J., Lincoln, D.T., Barnard, R., Wilcox, J.N.: Localization and ontogeny of growth hormone receptor gene expression in the central nervous system. Brain Res 1993; 74: 225-233.

11. Noel, F., Tofilon, P.J.: Astrocytes protect against Xray induced Neuronal toxicity in vitro. Neuroreport 1998, 9: 1133- 
1137.

12. Noguchi, T.: Effects of growth hormone on cerebral development. Morphological Studies. Horm Res 1996; 45: 517.

13. Peña, L.A., Fuks, Z., Kolesnick, R.N.: Radiationinduced apoptosis of endothelial cells in the murine central nervous system. Protection by fibroblast growth factor and sphingomyelinase deficiency. Cancer Res 2000; 60: 321327.

14. Prieto, I., Gómez de Segura, I., García Grande, A.: Morphometric and proliferative effects of growth hormone on radiation enteritis in the rat.Rev Esp Enferm Dig 1998; 90: 169-173.

15. Ross, G.M.: Induction of cell death by radiotherapy. Endocrin Related Cancer 1999, 6: 41-44.

16. Schultheiss, T.E., Kun, L.E., Ang, K.K., Stephens, L.C.: Radiation response of the central nervous system. Int J Radiat Oncol Biol Phys 1995; 31: 1093-1112.

17. Shoba, L., An, M.R, Frank, S.J., Lowe, Jr. W.L.: Developmental regulation of insulin-like growth factor-I and
2007; 18: 89-94

growth hormone receptor gene expression. Mol Cell Endocrinol 1999; 152: 125-136.

18. Vázquez, I., Gómez de Segura, I., García Grande, A., Escribano, A., De Miguel, E. Protective effect of enriched diet plus growth hormone administration on radiationinduced intestinal injury and on its evolutionary pattern in the rat. Digestive Disease \& Sciences 1999; 44: 2350-2358.

\section{Agradecimientos}

Este trabajo ha sido financiado por el FIS (PIO21225) y AESLEME

Isla, A.; Budke, M.; García-Grande, A.; Gómez de la Riva, A.; Morales, C.; Rey, J.: Radioprotección de la médula espinal mediante la hormona de crecimiento (GH). Estudio experimental en ratas. Neurocirugía 2007; 18: 89-94.

Correspondencia postal: Dr. Alberto Isla Guerrero. Servicio de Neurocirugía. Hospital Universitario La Paz. Paseo de la Castellana 261. 28046 Madrid 